\title{
A Survey of Energy Conservation Mechanisms for Dynamic Cluster Based Wireless Sensor Networks
}

\author{
RABIA NOOR ENAM*†, MUHAMMAD TAHIR*, REHAN QURESHI**, AND SHAKIL AHMED* \\ RECEIVED ON 19.11.2016 ACCEPTED ON 29.05.2017
}

\begin{abstract}
WSN (Wireless Sensor Network) is an emerging technology that has unlimited potential for numerous application areas including military, crisis management, environmental, transportation, medical, home/ city automations and smart spaces. But energy constrained nature of WSNs necessitates that their architecture and communicating protocols to be designed in an energy aware manner. Sensor data collection through clustering mechanisms has become a common strategy in WSN. This paper presents a survey report on the major perspectives with which energy conservation mechanisms has been proposed in dynamic cluster based WSNs so far. All the solutions discussed in this paper focus on the cluster based protocols only.We have covered a vast scale of existing energy efficient protocols and have categorized them in six categories. In the beginning of this paper the fundamentals of the energy constraint issues of WSNs have been discussed and an overview of the causes of energy consumptions at all layers of WSN has been given. Later in this paper several previously proposed energy efficient protocols of
\end{abstract} WSNs are presented.

Key Words: Wireless Sensor Networks; Dynamic Clustering protocols; Energy Conservation Mechanisms.

\section{INTRODUCTION}

1 dvancements made in communication technologies have enabled development of low-cost, low-power, small sized and multifunctional sensor nodes for WSNs. On the other hand, this has created some very significant resource constraints in WSNs for example limited buffer size, bandwidth and power supplies of SN (Sensor Nodes). These constraints create challenging issues when designing the hardware, software and architectures of
WSNs. Amongst them, energy or power limitations are vital problems as in most cases batteries of the $\mathrm{SN}$ are not rechargeable or replaceable. Since the lifetime of a sensor node is based on its energy level therefore minimizing the energy consumption of each node to prolong the lifetime of the network is very crucial.

In recent years there has been progress in the field of EH (Energy Harvesting). EH is the accumulation of energy

$\dagger$ Corresponding Author (E-Mail: afaq_rabia@yahoo.com)

* Department of Computer Engineering, Sir Syed University of Engineering \& Technology, Karachi.

** Department of Computer \& Software Engineering, Bahria University, Karachi.

This is an open access article published by Mehran University Research Journal of Engineering and Technology, Jamshoro under the CC by 4.0 International License. 
from outer sources for example solar power, wind power, vibration etc. In many cases the energies harvested are in very small amount and are generated and stored for short durations in SN. Therefore, the need of energy conservationor cautious utilization of energy should not be underestimated. In these cases, careful utilization of energy will not only help in increasing the node's lifetime but also will guarantee the working of nodes at times when it is unable to harvest the energy for itself. In this paper we have presented an extensive survey of several previously proposed energy efficient protocols of WSNs. Our focus is on cluster based protocols of WSNs only A lot of paper has been presented on efficient energy conservation mechanisms in cluster based WSNs. But this paper has categorized all the existing mechanisms in 6 different types of energy conservation means. While each category has been explained in a generalized form including multiple protocol examples. Some relevant existing solutions along with distinct energy conserving proposed protocols have been discussed in this paper.

Remaining paper is organized as follows: In section 2 the fundamentals of the energy constrain issues of WSNs have been given and an overview of the causes of energy consumptions at all layers of WSN has been discussed. Later in section 3, different existing energy conservation solutions have been discussed in detail and have been categorized accordingly. In the end in section 4 we have concluded our discussions.

\section{BACKGROUND}

In WSN there are several reasons that cause necessary and/or unnecessary utilization of energy. Energy consumptions occur at all layers of networking; physical layer, MAC layer, network layer and is also dependent on the application requirement. Many approaches have been adopted by the researchers in the past to conserve the scarce node energy as much as possible such as [1-2]. In this section we give an overview of causes of energy utilization in WSNs.

\subsection{Energy Consumption at MAC Layer}

MAC layer provides the accessibility to communication channels and there are two basic strategies used for this purpose: contention based and schedule (contention free) based [3-4].

Most of the WSN protocols use combination of these two strategies on their MAC layer. For example, in LEACH (Low Energy Adaptive Clustering Hierarchy), the cluster setup is done in non-persistent CSMA (np-CSMA) mode while data transmissions are done in TDMA (Time Division Multiple Access) scheduled slots [5]. Similarly, WiseMAC use preamble sampling technique to minimize the power consumed when listening to an idle medium while all nodes are defined to have their communication channels.

Some common reasons for energy consumptions in MAC layer are:

(a) Idle listening, in anticipation of possible arrival of frames. This is one of the major source of energy consumption. Most of the time during idle listening nodes receive data frames that are destined for other nodes.

(b) Transmission of more than one data frame in a network causes collisions. With large amount of nodes, chances of collisions and retransmission of data frames increase thereby increasing the energy consumption. Although few data frames can be recovered through a Capture effect. In capture effect the strongest transmitter may capture an intended receiver and many data frames may survive despite the collisions on channel but there are a number of requirements that have to be fulfilled for this kind of data recovery. 
(c) Transmission of control frames to initiate, acknowledge and re-establish the connections consume energy. For example, the overheads of CTS (Clear to Send), RTS (Ready to Send) and ACK (Acknowledgement) frame.

Another reason for energy consumption on MAC layer is over-emitting of frames. The major reason of over-emitting is the non-synchronization among the sender and receivers in a wireless medium. A node transmits a message when the destination is not ready to receive it.

\subsection{Energy Consumption at Network Layer}

Like other wireless networks, in WSNs the job of network layer is to obtain suitable routes from source nodes to destination. In WSN the optimal routes may be selected based on the number of hops, required transmission power and available energy on the relaying nodes. These parameters make the job complex, especially in multi-hop communications [4]. In network layer some common reasons of energy consumptions are:

(a) Redundant data transmissions in the network. Usually in WSNs more than required numbers of nodes are deployed. Having large number of nodes give better coverage and robust fault tolerance in the network, however it also generates redundant data flows in the network.

(b) In multi-hop relaying of data towards the sink, the SN around the sink create bottle necks and their energy depletes at much higher rates than the nodes farther away from the sink. This is also known as Hot-Spot problem [6].

(c) Transmissions of control packets for topology controls. The overheads in path set-ups in tree based protocols, CH (Cluster Head) selections and cluster set-ups in cluster based protocols are one of the major sources of the network energy utilizations. Control packets in a cluster formation phase the control packets transferred in broadcasting $\mathrm{CH}$ advertisements, nodes association requests and TDMA schedule distributions are examples of energy utilization in cluster formation phase.

\subsection{Communication vs. Computation Energy}

Communication is the dominant factor for power consumptions in WSNs. In most cases it has been observed that energy consumed in communication of data is many times higher than the energy consumed in processing of data. Thus omitting as much network traffic as possible is a substantial task in designing WSN. The fact that a SN consumes significantly less energy for information processing than for communication, makes it important to concentrate more on energy efficient communication rather than energy efficient computation.

In an active network at any instant, a node remains in one of the four states; Transmission, Reception, Idle listening or Sleep states. The basic energy model given in Equations (1-3) define the amount of energy consumed in these states. Here $E_{T X}$ is the energy consumed in transmission of data, $E_{R X}$ is the energy consumed in reception of data and $\mathrm{E}_{\mathrm{IX}}$ is the energy consumed in idle listening. $\beta$ is the ratio of reception and idle listening energy state. $\mathrm{E}_{\text {elec }}$ and $\mathrm{E}_{\mathrm{amp}}$ are the energies consumed in electronic circuitry and the amplification power of the node, respectively. $\mathrm{E}_{\text {elec }}$ and $\mathrm{E}_{\text {amp }}$ are kept constant for a given node type.

$\mathrm{E}_{\mathrm{TX}}=\mathrm{E}_{\text {elec }} \times \mathrm{k}+\mathrm{E}_{\text {amp }} \times \mathrm{k} \times \mathrm{d}^{2}$
$\mathrm{E}_{\mathrm{RX}}=\mathrm{E}_{\text {elec }} \times \mathrm{k}$
$\mathrm{E}_{\mathrm{IX}}=\beta \times \mathrm{E}_{\text {elec }} \times \mathrm{k}$ 
It can be seen that energy consumed in the transmission of data, $\mathrm{E}_{\mathrm{TX}}$ is dependent heavily on two factors: size of the data packet $(\mathrm{k})$ and the distance between the source and destination (d). Whereas, energy consumed in the reception of data, $\mathrm{E}_{\mathrm{RX}}$ is also dependent on the size of data. Size of data also affects the idle listening energy $\mathrm{E}_{\mathrm{IX}}$ since it is equivalent to $\beta$ times of $\mathrm{E}_{\mathrm{RX}}$. The usual range of $\beta$ lies between $0.5-0.8$. Sensors in sleep mode are same as in shut down state except that they keep their low-power timers on to wake up the sensor at later time. Therefore, a nominal fraction of the energy is utilized in the sleep mode.

In general, the major source of energy consumption is the transmission of large amount of data or information collected by the sensor nodes to the sink. We need to create energy-aware design for operations of all aspects of sensor networks from underlying hardware platform to network protocols to the application software. A wellstructured design methodologyat each level is required to minimize the redundant data transmissions and maximize the network lifetime.

\section{ENERGYCONSERVATIONSOLUTIONS FOR CLUSTER BASED NETWORKS}

Energy constraints in WSN nodes induce limitations in several aspects of networking for instance data sensing rate, communication rate, network lifetime and size and weight of SN. Many approaches have been suggested in the past to reduce energy consumptions in cluster based protocols. There are MAC layers that allow some
$\mathrm{SN}$ to remain in sleep mode for a suitable period of time while keeping other nodes in active mode for the sensing and communication tasks. Also MAC layers provide transmission schedules, keeping retransmissions of data as low as possible. Other ways include routing and data collection protocols that reduce the communication range among the nodes and reduce the control messages used in routing and path set-ups in the network [7]. Energy efficient routing techniques have been proposed to help route data within the sensor network with the aim of minimizing duplicate packets and number of hops, which leads to conserving energy of the network [8-9]. We have categorized previously proposed cluster based protocols according to their energy conserving mechanisms. Table 1 gives an overview of the classification of these protocols.

\subsection{Data Aggregation and Compression Solutions}

To get a better coverage and robust fault tolerance in the network usually more than required numbers of nodes are deployed in WSN. Since the cost and sizes of SN are trivial, therefore it is possible to have redundant nodes in the network and these spare nodes also generate redundant data flow in the network. To reduce the redundant data transmissions several data collection methods have been used [10-11]. One of the most commonly used methods is the efficient aggregation of data in the network.

TABLE 1. MERITS AND DEMERITS OF LOAD BALANCING THROUGH UNIFORM CLUSTERING

\begin{tabular}{|c|c|c|}
\hline Protocols & Merits & Demerits \\
\hline Centrally Controlled Protocols & $\begin{array}{c}\text { The central node, knowing the global information } \\
\text { of nodes forms more uniform sized and energy } \\
\text { efficient clusters }\end{array}$ & $\begin{array}{c}\text { In order to provide each nodes status to sink, } \\
\text { extra overhead of control packets } \\
\text { communications required }\end{array}$ \\
\hline Grid-Cluster Protocols & $\begin{array}{c}\text { CH selection decisions are distributed among the } \\
\text { nodes themselves. Therefore, it can work well in a } \\
\text { large scale network }\end{array}$ & $\begin{array}{c}\text { Total network energy consumed is high. Because } \\
\text { the selections of the CHs in grid-cluster based } \\
\text { protocols are based only on the energy levels of } \\
\text { the nodes within the grid not with respect to the } \\
\text { whole network }\end{array}$ \\
\hline Radio Range Based Protocols & $\begin{array}{c}\text { The sizes of clusters can be controlled by } \\
\text { controlling the radio frequency ranges of CHs }\end{array}$ & $\begin{array}{c}\text { Due to several multipath interferences in } \\
\text { communication channels precise formation }\end{array}$ \\
\hline
\end{tabular}


Although the size of an individual piece of data collected from a single node is insignificant but in large scale networks data collected from different sources are forwarded towards the sink in an aggregated form. This can increase the size of payloads; therefore, data compression techniques are applied to reduce the overall energy consumptions.

In this section a brief overview of different types of data aggregations is given. Since in many cases data compression has been considered as a part of aggregation process therefore some compression techniques including the conventional compression techniques are also discussed.

\subsubsection{Data Aggregation}

Data aggregation process is based on the methods of gathering and compressing data at an aggregation point in a network [12-13]. The basic motivation behind any aggregation process is to suppress the redundancy in the collected data and to reduce the overall transmission cost of the network. In a cluster based network it is the duty of the CHs to aggregate the data before forwarding it to the sink [14].

We have classified the data collection methods into different categories as shown in Fig. 1. In order to reduce the redundant transmission of data, these methods have been used jointly or separately in different protocols of WSN.

Data Suppression: Is the simplest form of decreasing the amount of data in the network. This method mostly exploits the temporal, spatial and/or semantic correlations among the data. The samples taken from nodes which are in close proximity to each other tend to have similar values which are called spatially correlated data. In periodic sampling procedure the samples taken from a SN within a short period of time tend to have similar value between each pair of consecutive observations. These data are called temporally correlated data. In semantic correlations the data are reported by multiple sensors due to the semantics of the query generated by the sink or some other monitoring sources. Based on the degree of correlation many of the nodes can be kept in sleep mode or prohibited to transmit their data at the same time, hence reducing unnecessary flow of data in the network.

Packet merging is also a very simple method of accumulating data in one place without reducing the data sizes. The packets coming from different sources are merged into one packet at a collection point or an aggregation point, without processing the value of data [15]. In these cases, the packet sizes at the aggregation points are assumed to be big enough to accommodate large amount of data.

Generally, in literature, there have been two perspectives of looking at the data aggregations in a WSN. One is by the means and methods used to route and forward data through the best path to the sink while collecting data on the way. This method is usually called as In-network

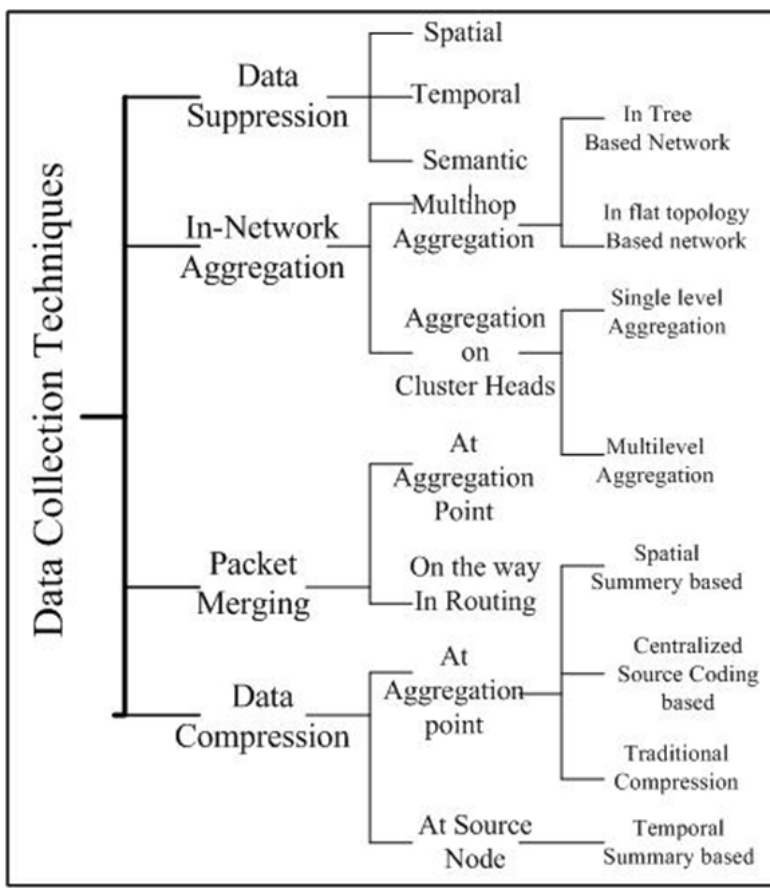

FIG. 1. SUMMARY OF DATA COLLECTION TECHNIQUES 
aggregation. The data collections and suppressions are done along with the multi-hop data forwarding, for example the methods followed in Directed Diffusion [16-18]. For instance in [18] the authors have tried to minimize the network energy consumptions by combining the processes of compression sensing based aggregation and data routing. Compression sensing is a newly developed signal processing technique which gives full recovery of signals at the receiver side. The basic idea behind these methods is that the routing protocols in a WSN can use the natural gradients of correlated data as an important attribute to forward the query towards source or send the collected data back to the sink.

Another way of looking at the data aggregation process, that has been discussed in literature widely is the methods of suppressing or compressing the data at an aggregation point (for example CHs in cluster based networks) such as aggregation processes described in [12,14]. The basic idea behind these methods is to suppress the redundancy in the collected data to reduce the overall transmission cost of the network. Since spatial and temporal correlations among the sensed data are one of the basic characteristics of WSN, therefore, this unique feature of WSN has been extensively exploited for the purpose of data aggregation and compression [13].

\subsubsection{Summary Based Aggregation}

Taking mean, median and max/min values of a set of collected data are the simplest form of data compression.This method is also called Summary based aggregation.These methods take minimum amount of bits that is why many of the WSN protocols have acquired it for their data aggregation processes [19-20]. Since in these methods there is a single data value that represent multiple sensors readings, therefore, these methods can give the highest degree of inaccuracy. These data compressing methods cannot give good data representation.

\subsubsection{Traditional Data Compression}

There have been many traditional compressing schemes, compared and analyzed for different types of sensor networks, such as Huffman coding, Adaptive coding and Delta coding. Although these algorithms are loss less and provide light compressions for linearly varying data values, but they require a heavy code book for the recovery of data at the receiver. These schemes suit well for limited sensor data types and cannot be applied to a WSN consisting of multiple data types which have nonlinear behavior [13].

\subsubsection{Centralized Source Coding Based Data Compression}

Centralized source coding based aggregation is a method where the compression is done at the aggregation points rather than on the data originating nodes. In a large scale cluster based WSN with spatially correlated nodes, the centralized source coding algorithm, suits more to the data collection strategies and give efficient data aggregation environments However, there are some cluster based schemes that com- press the data at their originating sources instead of their $\mathrm{CHs}$, for example in [13]. In our view, considering the optimal size of packets in a WSN and the general size of a sensed data, the need of compressing data at the originating source is not much fruitful. In order to reduce the redundant data transmissions in the network, either the SN should transmit their data packets otherwise they should be kept in sleep mode for a considerable amount of time.

Some of the centralized source coding based protocol that has been proposed so far is RDAC [21]. In these methods the data are collected at the CHs where aggregation with compression algorithms is performed.

In RDAC [12], the authors have proposed a centralized source coding based correlation aggregation algorithm

Mehran University Research Journal of Engineering \& Technology, Volume 37, No. 2, April, 2018 [p-ISSN: 0254-7821, e-ISSN: 2413-7219] 
that works on RD (Rate Distortion) method. RD method addresses the problem of finding the minimum number of bits per symbol that should be communicated over a channel, such that the source's signal can be approximately reconstructed at the receiver without exceeding a given distortion. In RDAC the correlation factor is based only on the distances between the nodes. Therefore, at each $\mathrm{CH}$ for its $\mathrm{N}$ associated nodes a computation of correlation matrix of size NxN and its Eigen vector are required. The correlation levels among the nodes are provided by the sink node. It is good for computing the $\mathrm{NxN}$ matrix and its Eigen vector once but would not be feasible in a dynamic cluster based network where the clusters are formed repeatedly. Almost the same method of forming correlation matrix has been adapted in [21].

In 3D-DCT (Discrete Cosine Transform) technique has been used which exploits the spatial-temporal correlation for the aggregation of data. In this method the data are transferred into uncorrelated frequency domain coefficients at an aggregation point.A 3D-Zigzag sorting algorithm makes sure that the aggregation point transfers frequency coefficients from lower frequencies that contain the main energy of the original data to higher frequencies [22].

The aggregation techniques proposed in $\operatorname{RDAC}[12,21]$ and 3D-DCT [22] do not consider some important factors of clustering like the size of clusters or the amount of data collected from a cluster and the packet sizes available at a $\mathrm{CH}$ for data aggregations. The ADiDA (Adaptive Differential Data Aggregation) technique [23] is also a centralized source coding based aggregation scheme. ADiDA considers the aspects like correlation among data and sensing ranges of nodes, but beside these aspects the main parameters that regulate the adaptive nature of ADiDA are the amount of data collected from a cluster and the size of packet available for the aggregation of these data. Both of these parameters can vary at run time therefore the proposed aggregation method is adaptive to the variations of these two parameters whereas it gives a trade-off for the distortion level in the aggregated data. Based on the proposed method an optimal payload size has also been estimated. ADiDA is discussed in [23] in detail. Since amount of data in a cluster is dependent on the size of cluster in terms of number of nodes, therefore the efficiency of ADiDA is also dependent on the uniformity in the cluster sizes. In the following section we have discussed various uniform clustering mechanisms.

\subsection{Load Balancing through Uniform Clustering}

Usually in cluster based protocols of WSN, dynamic clustering is done to evenly distribute the consumption of energy among all nodes of the network. Dynamic clustering is a process in which the role of $\mathrm{CH}$ is rotated among different nodes and the clusters are formed repeatedly in the network. There are data collection rounds and in each round based on some criteria $\mathrm{CHs}$ are selected and subsequent clusters are formed in the network. There are several protocols like [19-20], in which the locations of the $\mathrm{CHs}$ are not considered in the $\mathrm{CH}$ selection criteria. As a result, in these protocols the selected $\mathrm{CHs}$ are not uniformly distributed in the region. For example, it can be seen in Fig. 2(a) (A snap shot of one of the rounds, taken from a simulation of LEACH protocol [24]) that all of the CHs formed are in the lower right corner of the network area. As a result the sizes of clusters, in terms of number of nodes per cluster, are highly variable as shown in Fig. 2(b).

The major drawback of unequal clustering is that it can create few large sized clusters in the network which require large sized payloads so that any amount of data can be aggregated into it. In the clusters that are spread on large

Mehran University Research Journal of Engineering \& Technology, Volume 37, No. 2, April, 2018 [p-ISSN: 0254-7821, e-ISSN: 2413-7219] 
regions of network, there can be several nodes which needto contact distantly located $\mathrm{CHs}$ to forward their data. For example, in clusters 1 and 2 of Fig. 2(b). Therefore, long distance intra-cluster communication can create high energy consumption in the transmission of data in the network. The SN in these clusters placed at far off distances provide less degree of spatial correlations with each other, therefore spatial correlation based data compressions cannot be used easily.

There are some solutions that are proposed to overcome the variations in cluster sizes of the network. These mechanisms can be divided into three categories: centrally controlled cluster formation protocols, grid-cluster based protocols and radio range based clusteringprotocols.

\subsubsection{Centrally Controlled Protocols}

In centrally controlled cluster formation protocols the clusters are formed dynamically but the process is controlled centrally by the sink. Examples of centrally controlled fixed cluster protocols are explained in section III-C and examples of centrally controlled dynamic cluster

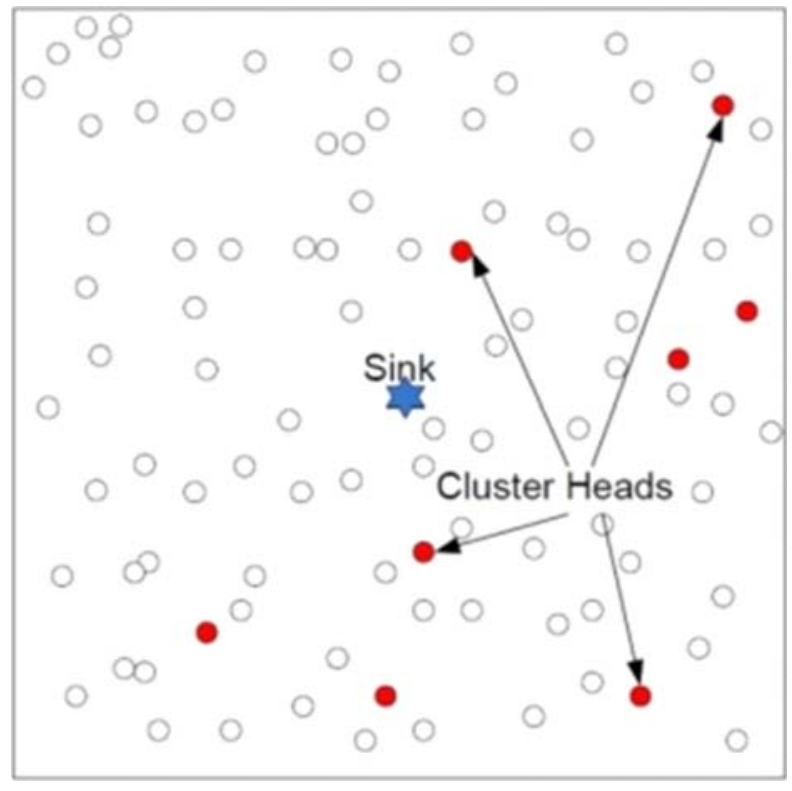

(a) CLUSTER HEADS SELECTED IN A ROUND based protocols are LEACH-C [24] and CEDCAP [25]. In LEACH-C [24] at the start of each cluster formation round, each node sends its residual energy level and location information to the sink node. The sink node selects CHs and builds clusters using the simulated annealing algorithm. This way the sink, knowing the global information of nodes forms more uniform sized and energy efficient clusters.

In Base-Station Controlled Dynamic Clustering Protocol during the cluster setup phase of each round all nodes send their current energy status to the sink. The sink chooses a set of $\mathrm{CH}$ based on their energy levels. To get clusters with approximately equal sizes, the sink runs the cluster splitting algorithm iteratively. In each step of iteration, the algorithm selects the $\mathrm{CHs}$ which are uniformly placed by maximizing the distances between the selected CHs. To form a cluster, the nodes are associated to the $\mathrm{CHs}$ based on the closest distance. After the nodes association the extra nodes are handed and taken over between the two splitted clusters in order to balance the size of the clusters. This whole process is centrally controlled by the sink node.

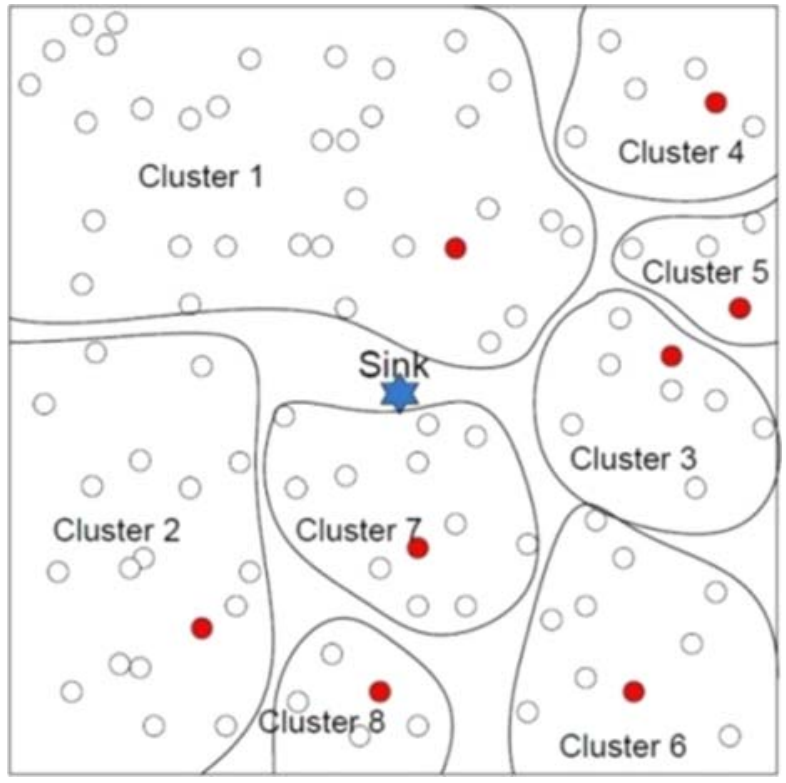

(b) FORMATION OF IRREGULAR SIZED CLUSTERS

FIG. 2. DYNAMIC CLUSTER BASED WSN 
Similarly, in CEDCAP (Cluster-Based Energy-Efficient Data Collecting and Aggregation Protocol) [25] after receiving the knowledge of energy levels and locations of all the nodes in the network, the sink selects the $\mathrm{CHs}$ and their associated nodes. Besides selecting the $\mathrm{CHs}$ and associated nodes of the cluster, the sink also sends the TDMA schedule to the nodes of the clusters for the transmissions of data. The TDMA calculated by the sink are scheduled according to the node degree control for each cluster. Based on the node degree control a set of nodes in each cluster are kept dormant for some period of time hence reducing the redundant data transmission in the network. The basic requirement of a centrally controlled cluster formation protocol is that in order to provide each node's status to sink, either every node should have a direct contact to the sink, if not, extra overhead of control packets and communications are used in the network. Whereas in a network with a large number of nodes or if nodes are placed at far off places this kind of approach require extra multi-hop communications to be applied.

\subsubsection{Grid-Cluster Based Protocols}

In grid-cluster based protocols unlike centrally controlled cluster formation protocols, the $\mathrm{CH}$ selection decisions are distributed among the nodes themselves. Therefore, it can work well in a large scale network. The fundamental idea of the grid-cluster based protocols of WSN is to divide the whole network area into equal sized virtual grids where each grid is considered as a cluster with one $\mathrm{CH}$ in each cluster $[9,26,27]$. The role of $\mathrm{CH}$ is rotated among different nodes within a cluster. Fig. 3 depicts the grid-cluster method where every grid contains one $\mathrm{CH}$. The main characteristics of the grid-cluster based protocols are that a grid is considered as a whole cluster, where the clusters are fixed and not dynamic. Due to unequal and random deployment of nodes the size of clusters (In terms of number of nodes) does not get uniformly distributed still. Authors in [26] have tried further to even out this irregularity, such that after setting one $\mathrm{CH}$ in each grid, the sizes of clusters are adjusted by handing and taking over extra nodes to and from the neighbor grids. These are done through a centralized control system and require an extra overhead of control packets which consumes extra energy and delay in the system.

A grid-cluster based method gives substantially reduced irregularity in the distributions of $\mathrm{CHs}$ and in the variationsof the cluster sizes, but the total network energy consumed in these protocols are very high compared to a dynamic cluster based protocol. This is because the selections of the CHs in grid-cluster based protocols are based only on the energy levels of the nodes within the grid not with respect to the whole network. The second reason of high energy consumption is that the clusters are not formed on the basis of the distances of nodes to their closest CHs. Irrespective of the distance a node will always have to associate to the $\mathrm{CH}$ belonging to its own grid. For example in Fig. 3 in grid number 1 the nodes in the lower right corner of the grid will have to transmit their data to the distant $\mathrm{CH}$ in their grid whereas they had two closer CHs available in grid number 2 and 5.

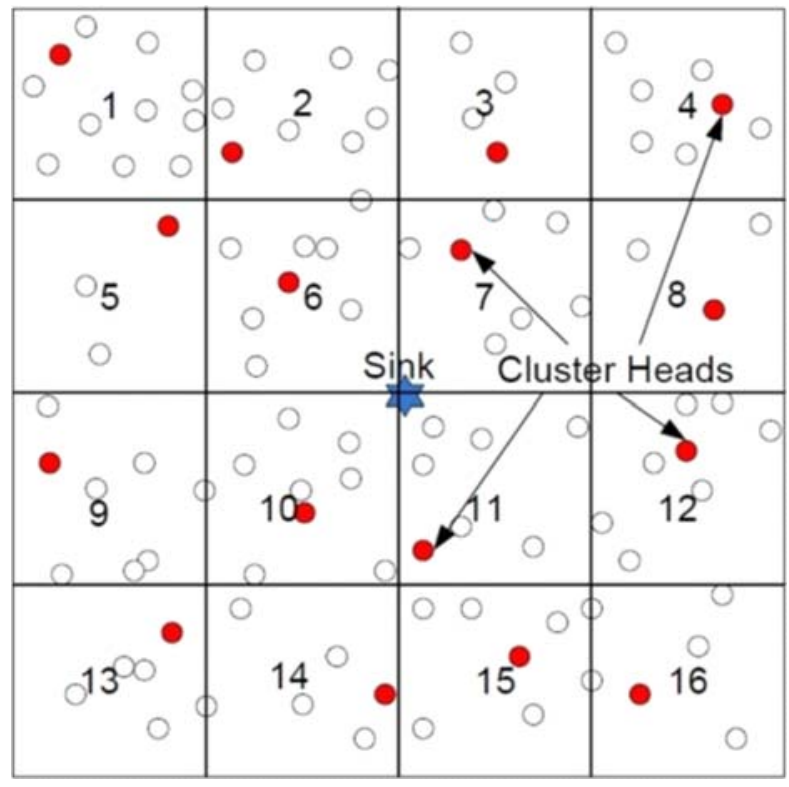

FIG. 3. GRID-CLUSTER BASEDWSN

Mehran University Research Journal of Engineering \& Technology, Volume 37, No. 2, April, 2018 [p-ISSN: 0254-7821, e-ISSN: 2413-7219] 
It can be noted that the methods described in Grid based clusterings like in [27] require location information to form equal sized clusters in the network. These methods can give optimum results if the nodes are deployed equidistantly. In case of randomly deployed nodes the results can vary since in this method the clusters are formed according to the geographical location points and not the spread of the nodes in the field.

In [28] we have proposed a mechanism named as DUCA (Dynamic Uniform Clustering Algorithm) Protocol which can provide even distribution of CHs and clusters by implementing an energy efficient usage of virtual grids in the network. Unlike grid-cluster protocols discussed in this section, in DUCA a grid is not taken as a cluster rather clusters are formed on the basis of distances of nodes to their nearest CHs. This has given a better distribution of energy consumption with respect to the entire network.

\subsubsection{Radio Range Based Protocols}

In this approach CHs through controlled range of radios broadcast their advertisements therefore, the nodes beyond the range cannot listen and cannot join the cluster hence in this manner the sizes of clusters are controlled. For example, the methods proposed in VCCBC (Virtual Co-centric Circle Band Based Clustering) [29]. In VCCBC, initially the network area is divided into concentric circles of equal gap. The width of this gap is assumed to be $\mathrm{R} / 2$ meters where $\mathrm{R}$ is the maximum transmission range of a node. These virtual concentric divisions of area are used to distribute the $\mathrm{CHs}$ evenly and equidistantly within each concentric circle. After $\mathrm{CH}$ selections, the $\mathrm{CHs}$ broadcast their advertisements within $\mathrm{R} / 4$ range. The remaining nodes get associated to their nearest $\mathrm{CHs}$ based on the signal strengths of $\mathrm{CH}$ advertisements. Once the clusters are formed, the role of $\mathrm{CH}$ is rotated among the nodes of the same cluster. The authors have calculated the energy consumption during $\mathrm{CH}$ selection and rotation phases. To minimize the energy consumption in $\mathrm{CH}$ rotation, the $\mathrm{CH}$ rotation takes place uniformly after an optimal number of rounds. The optimal numbers of rounds are precalculated based on the total energy consumed per round in the cluster and the total initial energy of that cluster.

In short, the proposed method in VCCBC [29] rely on the radio ranges of the nodes which are varied during the cluster formationstoform equidistant and equal sized clusters in the network. Once the clusters are formed the role of CHs are rotated among the nodes of the same cluster. Since the estimation of distances based on radio ranges can give high variances due to different environmental interferences and diverse channel conditions, therefore the formation of equal sized clusters cannot be guaranteed in these protocols also.

To improve the efficiency of our proposed aggregation algorithm ADiDA proposed in [23] uniform clustering was required. Some of the methods discussed in this section can provide very good results in terms of uniform clustering but require extra amount of energy to form equal sized clusters in the network. In this [28]we have proposed a mechanism named DUCA which can give better results in terms of uniformity of clusters and conservation of energy in the network, discussed in [28]. We have further reduced the sizes of clusters by exploiting the redundant and overlapped sensing ranges, which occur due to the random placements of sensor nodes in the network. Table 1 gives a comparison of the uniform clustering protocols discussed in this section.

\subsection{Reduction in Cluster Setup Overhead}

In large scale WSN, the data collection through dynamic clustering was introduced by Heinzelman [24]. In dynamic clustering, network lifetime is divided into data collection rounds, where in each round the job of $\mathrm{CH}$ is assigned 
rotationally to different nodes and subsequent clusters are formed repeatedly in the network. The idea behind this process is to evenly distribute the consumption of energy among all the nodes. To achieve this, a mechanism called re-clustering is done in each round.

Re-clustering is a process where in every data collection round, different set of nodes become $\mathrm{CHs}$ and these $\mathrm{CHs}$ form clusters with their neighboring nodes. Since CHs consume more energy than the regular nodes, the job of $\mathrm{CH}$ is handed-over to different nodes in different rounds. But efficient selection of $\mathrm{CHs}$ and subsequent formation of clusters always need exchange of control packets which consequently consume extra network energy. There are some protocols proposed in the past which have aimed to overcome these extra network energy consumptions by avoiding the re-clustering in the network.

The most common method to avoid re-clustering is to fix the clusters once they are formed and rotate the job of $\mathrm{CH}$ among the nodes within a cluster [29]. The essential requirement of one-time-clustering approach is that the clusters formed should be of uniform sizes so that the load is evenly balanced among all the clusters. One of the approaches used is the centralized control system for example LEACH-F [24]. LEACH-F is a modified version of LEACH. In LEACH-F, initially all nodes provide their location information and energy levels to the sink. Based on this information the sink divides the network into required number of clusters. Once the clusters are formed, they remain fixed for the remaining lifetime of the network. However, the role of $\mathrm{CH}$ is rotated among different member nodes of the same cluster. Similar to LEACH-F, there are other protocols that require a centralized clustering approach such that all nodes need to communicate directly or indirectly to the sink. This method can give a fair energy saving in the network but, in a large scale network, may give difficulty or overheads to the far off nodes to get a direct communication or repeated indirect communication to the sink.
To conclude we can say that re-clustering is a process used in dynamic cluster protocols to evenly distribute the consumption of entire network's energy. On the other hand re- clustering itself consumes some extra energy of the network. In some of the previously proposed methods the idea of conserving the energy during the $\mathrm{CH}$ setup or cluster formation phases is avoided by forming the clusters only once in the networks lifetime and then rotating the role of $\mathrm{CH}$ among different nodes of the same cluster. In other words, they have completely avoided the process of re-clustering in the network. Since these methods do not provide a periodic synchronization among all the nodes of the network, therefore a perturbed environment can be produced in the network after some time period. For example; in the cases of nodes failure or changes in channel conditions. Load balancing through uniform clustering is also essential for one-time-clustering approach and in case of $\mathrm{CH}$ failure there should be some centralized control system within a cluster that can handover the role of $\mathrm{CH}$ to another node.

In [19] we have proposed a dynamic clustering mechanism called RR (Round Rotation) method which can reduce up to $80 \%$ of the overhead energy consumed in cluster setups only. Unlike fixed clusters based approaches, the basic feature of RR method that differentiate it from existing solutions is that it can work for dynamic cluster based protocols.

\subsection{Energy Efficient Inter and Intra Cluster Routing}

One of the basic features of WSN that distinguishes it from other traditional wireless networks is its convergecast based data forwarding. Converge-cast is a process of collecting and forwarding data from all or a set of SN towardsthe sink (many to one communication). Since in WSNs there are many sources and only one final destination (sink) of data, therefore it employs converge- 
cast approach in all of the data routing protocols. This feature comes with a specific traffic pattern in the network as shown in Fig. 4. Due to the energy constrained nature of sensors and the requirements set forth by the monitoring applications, energy and latency minimization are the important requirement in WSNs. To achieve an optimal and energy aware routing in such networks can be a challenging task.

Basic aim of routing in WSNs is to conserve the energy of network by minimizing the duplicate packets and number of hops in a path. There are two types of routing in cluster based WSNs: intra-cluster routing and inter-cluster routing. Intra-cluster routing is done within the clusters having large number of nodes that are dispersed in wide regions of the network. In these clusters, energy efficient routing is required to collect data from the entire cluster on its $\mathrm{CH}$. Intra-cluster routing is also based on convergecast approach because there are multiple sources and one destination that is $\mathrm{CH}$ of the cluster.

In inter-cluster routing the nodes or $\mathrm{CHs}$ are involved in multi-hop forwarding of data towards the sink. To improve inter-cluster routings many solutions have been proposed in the literature. Most of the routing and flow problems are formulated through SPT (Shortest Path Tree) or ST (Spanning Tree) problems. Examples are discussed below.

Minimum SPT-PSO [30] used PSO (Particle Swarm Optimization) approach to achieve optimal inter-cluster routing path for the optimization of network lifetime. PSO algorithm can generate new routes where energy levels are taken as fitness.

Value to judge different paths and choose best optimized path whose energy consumption is lesser than other routing paths. To construct $\mathrm{CH}$ based energy efficient routing tree in the network, the important factor that should be fulfilled is the uniform distribution of $\mathrm{CHs}$ in the network. Therefore, location based $\mathrm{CH}$ selection and cluster formation is necessary for this approach. Many locations based clustering approach has been used in previous works like LEACH-C [24] and GROUP [31]. In [32] before the formation of clusters, DAT (Data Aggregation Tree) is formed using $\mathrm{N}_{\mathrm{t}}$ nodes out of total $\mathrm{N}$ nodes in the network. These $\mathrm{N}_{\mathrm{t}}$ nodes collect the data from its neighboring nodes, aggregate the data and then route the data through the DAT towards the sink. To make sure that all other non-tree nodes can find at least one $N_{t}$ node in its close proximity, the nodes of DAT are well spread and uniformly distributed in the entire region. For the construction of DAT, initially each node should know its node ID and all of its one-hop and two-hop neighbors. This is achieved by initially flooding the network by Hello packets from all the nodes. Besides giving energy efficient routing, DAT can collect and do in network aggregation of data collected from the entire network. In all of the works mentioned above, to get a minimum energy routing, shortest path tree has been constructed using CHs and/or other nodes explicitly or implicitly. Different link cost approaches have been adapted for example hop count, residual energy of path, average energy of nodes constructing the path. In majority of the cases the main aim is to use the least energy consuming paths within the network for the transmission of data from SN to sink.

\subsection{Cluster Head Selection Criteria}

In research literature it is generally found that cluster based topology of WSN enables improved energy efficient data collection and routing mechanisms compared to other topologies. LEACH [24] is the pioneer in cluster based protocols that have proposed the concept of dynamic clustering. Dynamic clustering is a process in which the data are collected in rounds and in each round new sets of cluster with different nodes as $\mathrm{CHs}$, are formed. The main idea of LEACH that has made it popular is its fair selection of $\mathrm{CH}$ with respect to the entire number

Mehran University Research Journal of Engineering \& Technology, Volume 37, No. 2, April, 2018 [p-ISSN: 0254-7821, e-ISSN: 2413-7219] 
of nodes in the network. This gives an even distribution of energy consumption among all the nodes of the network. This idea of LEACH became so popular that it triggered many researchers to work further on it. Hence there have been more than 29 descendants of LEACH protocols that have been proposed so far [29]. Many of these modified versions of LEACH protocols have tried to further improve the $\mathrm{CH}$ selection mechanism of $\mathrm{LEACH}$ protocol. The main aims of these protocols were to optimally utilize the node's energy. For example, authors of Re-cluster-LEACH [33] have tried to improve the $\mathrm{CH}$ selection algorithm in LEACH by considering the density of nodes inside the cluster for $\mathrm{CH}$ formations, increasing the cluster based data fusion.

Unlike LEACH in which nodes choose their $\mathrm{CH}$ on the basis of distances, In LEACH-B [34] each SN chooses its $\mathrm{CH}$ as the ones which can provide least energy dissipation in the path between the final receiver and itself. It is a decentralized algorithm of cluster formation in which SN only know about their own position and position of final receiver and not the position of all SN. Like LEACH protocol in LEACH-B also the phases of $\mathrm{CH}$ selection, cluster formation and data transmission remains the same. Each $\mathrm{SN}$ chooses its $\mathrm{CH}$ by evaluating the energy dissipated in the path between final receiver and itself.

Besides the LEACH based protocol there are numerous other cluster based protocols that have improved the $\mathrm{CH}$ selection criteria, so that they could minimize the energy consumptions in the network. Many of these protocols have presented their own mechanisms of $\mathrm{CH}$ selection. For example, periodically selects CHs according to both the node's residual energy level and the node's proximity to their neighbors. As a result, the chances of $\mathrm{CH}$ selections increase in the region with more number of nodes giving more balanced distribution of energy consumption among all nodes. Another protocol called LGCA [35] uses game theory for the clustering of nodes where the nodes compete selfishly with its neighbor nodes which are in their communication ranges and suitable for the $\mathrm{CH}$ selection. In EEMA [36], the $\mathrm{CH}$ selection criterion has been enhanced by considering the centrality of the node and the proximity to the other neighboring $\mathrm{CHs}$ besides the residual energy of the nodes.

\subsection{Cluster Formation on the Basis of Distance to Sink}

The basic structure of WSN consists of multiple sources of data (SN) and one central point to collect the data (sink), therefore, the data transmissions in WSNs are generally based on many to one approach. With the multihop data relays in large scale WSNs, the many to one approach creates congestion at the nodes closer to sink. The larger the number of sensors in a network the more overloading of data transmissions will occur at the nodes that are neighboring the sink. Since the main causes of energy consumption in SN are the transmission and reception of data therefore, ultimately this causes early expiration of nodes closer to the sink. This problem is also called the Hot-Spot Problem [20]. Fig. 3 shows the Hot-Spot problem in a cluster based WSN where the CHs close to sink have to transmit and receive multiple times to forward the entire network's data to the sink. To resolve the Hot-spot problem, there has to be methods which should distribute the load of forwarding and routing the data evenly, among all the nodes in the network. Many approaches have been proposed in the past to solve this problem, but majority of them rely on unequal and distance to sink based sizing of clusters, for example [6,37].

For example, EECS [38], which was proposed for single hop clustered network, where dynamic sizing of clusters were made. The $\mathrm{CH}$ selection in EECS is done according

Mehran University Research Journal of Engineering \& Technology, Volume 37, No. 2, April, 2018 [p-ISSN: 0254-7821, e-ISSN: 2413-7219] 
to LEACH protocol but cluster formation is different from LEACH. In LEACH after $\mathrm{CH}$ selections, the clusters are formed on the basis of minimum distance of nodes to their CHs. In EECS, distance to sink based unequal clusters are formed such that farther the distance to sink, the smaller the clusters are formed. Therefore, conserving intra-cluster communication energy of CHs and using it for the long distance communications to sink. The basic thought behind this method is that the clusters at a greater distance from the sink requires more energy for transmission than those that are closer to sink, giving unequal distribution of energy in the networks. Thus main advantage of this algorithm is that it can provide reliable sensing capabilities at a larger range of networks for a longer period of time.

EEUC (Energy Efficient Unequal Clustering) [39] has concentrated on the creation of unequal clustering and multi-hop routing within its unequal clusters. The algorithm partitions all the nodes into unequal clusters such that clusters closer to the sink have smaller sizes than those farther away from the sink. The idea behind producing smaller clusters closer to sink is to conserve the energy consumed in intra-cluster communication so that the reserved energy could be used in multi-hop forwarding of other nodes data towards the sink.The CHs are elected based on localized competition. The RF range used for competition, decreases with the decrease of distance with the sink therefore smaller clusters is produced in the closer proximity of the sink. On the other hand for the purpose of inter cluster data routing, each $\mathrm{CH}$ chooses other $\mathrm{CH}$ as its relay agent, from its adjacent cluster, but on the basis of residual energy and the distance to the sink.

The protocol proposed in [37] has adopted a new approach of unequal clustering. In this protocol, a fuzzy logic system is used to determine each nodes chance of becoming $\mathrm{CH}$ and estimate the $\mathrm{CH}$ competence
radius.CHs are selected on the basis of each node's energy level, distance to sink and local density. For an efficient routing between $\mathrm{CHs}$ and sink, it uses Ant Colony Optimization method [37], whichcan solve the Hot-Spot problem by reducing and balancingthe energy consumption of CHs. Similar approach of unequal clustering has been adapted by UCA [20] which aiming to resolve the Hot-Spot problem and produce more uniform energy dissipation among the $\mathrm{CH}$ nodes.

In PEZCA (Power-Efficient Zoning Clustering Algorithm) [40] and E2GBR [41], a bit different approach of unequal clustering is used where instead of dividing the numbers of nodes into desired size of clusters the whole area is divided into sub regions. In PEZCA [40], the whole network region is divided into multiple fan shaped cells. Obviously here it has been assumed that the nodes are dispersed uniformly in the whole region. Therefore, such division of region produces smaller sized clusters closer to the sink compared to those farther away from the sink. Similarly, in E2GBR protocol [41], instead of fan shaped the authors have divided the region into unequal and non-uniform sized grids, small grids close to sink and larger grids farther from the sink. These grids are assumed as clusters. The sizes of grids are adjusted by varying the transmission range of nodes. Table 2 gives an overview of all the energy conservation protocols.

In all of the protocols mentioned above the basic intention is to reduce the transmission load on the nodes closer to sink and balance the overall consumption of energy in a cluster based WSN. For that, all of them have aimed to fairly distribute the consumption of energy between the inter-cluster and intra-cluster communication of data. Table 3 gives a comparison overview of all the types of energy conservation mechanisms that have been discussed in this paper.

Mehran University Research Journal of Engineering \& Technology, Volume 37, No. 2, April, 2018 [p-ISSN: 0254-7821, e-ISSN: 2413-7219] 
TABLE 2. ENERGY EFFICIENT MECHANISMS IN CLUSTER BASED PROTOCOLS OF WSN

\begin{tabular}{|c|c|c|}
\hline No. & Energy Efficient Mechanisms & Protocol Examples \\
\hline 1. & Energy efficient Inter or Intra cluster routing & $\begin{array}{c}\text { EEMA [36], DWEHC [27], Improved GCRP [26], } \\
\text { Minimum SPT-PSO [30] }\end{array}$ \\
\hline 2. & CH selection criteria & $\begin{array}{c}\text { Re-cluster-LEACH [33], LEACH-B [34], LGCA [35], } \\
\text { EEMA [36]. }\end{array}$ \\
\hline 3. & Reduction in cluster setup overhead & LEACH-F [24], Protocols proposed in [29] \\
\hline 4. & Load balancing in clusters & $\begin{array}{c}\text { LEACH-C [24], VCCBC [29], CEDCAP [25], and } \\
\text { Protocols proposed in [27] }\end{array}$ \\
\hline 5. & Distance to sink based cluster formation & UCA [20], Protocols proposed in [37] \\
\hline 6. & Data aggregation and compression solutions & $\begin{array}{c}\text { DACA[42], RDAC [12], Distributed clustering aggregation [14], } \\
\text { Spatial correlation based aggregation [17] }\end{array}$ \\
\hline
\end{tabular}

TABLE 3. MERITS AND DEMERITS OF ENERGY CONSERVATION MECHANISMS

\begin{tabular}{|c|c|c|}
\hline Energy Conservation Mechanisms & Merits & Demerits \\
\hline $\begin{array}{l}\text { Data Aggregation and } \\
\text { Compression Solutions }\end{array}$ & $\begin{array}{l}\text { Redundant data transmission and reception are } \\
\text { reduced saving energy consumption }\end{array}$ & $\begin{array}{l}\text { Extra computational load on nodes and/or CHs, } \\
\text { Accuracy of data may be lost }\end{array}$ \\
\hline $\begin{array}{l}\text { Load Balancing } \\
\text { Through Uniform, Clustering }\end{array}$ & $\begin{array}{c}\text { Load of data collection and forwarding is } \\
\text { evenly distributed among all nodes of } \\
\text { large scale network }\end{array}$ & $\begin{array}{c}\text { Extra computational load is required to form } \\
\text { uniform sized clusters. }\end{array}$ \\
\hline $\begin{array}{l}\text { Reduction in } \\
\text { Clustersetup Overhead }\end{array}$ & Over head of control messages are reduced & $\begin{array}{c}\text { May not optimally distribute energy consumption, } \\
\text { load among all nodes. }\end{array}$ \\
\hline $\begin{array}{c}\text { Energy Efficient } \\
\text { Inter and Intra Cluster, Routing }\end{array}$ & $\begin{array}{l}\text { Optimal utilization of each nodes energy } \\
\text { can be made }\end{array}$ & $\begin{array}{l}\text { Energy efficient path may not be least cost path in } \\
\text { terms of delay, bandwidth and security }\end{array}$ \\
\hline Cluster Head Selection Criteria & $\begin{array}{l}\text { Optimal utilization of, nodes energy } \\
\text { can be made }\end{array}$ & $\begin{array}{c}\text { Due to many other factors right selection of CHs } \\
\text { may not be made causing its energy depletion } \\
\text { faster }\end{array}$ \\
\hline $\begin{array}{l}\text { Cluster Formation on the Basis of } \\
\text { Distance to Sink }\end{array}$ & Hot-Spot problem can easily be solved & $\begin{array}{c}\text { May not be suitable for various application } \\
\text { requirement }\end{array}$ \\
\hline
\end{tabular}

\section{CONCLUSION}

In this paper initially we have discussed the reasons and factors that generally affect the energy constraints of WSN. Various aspects of cluster based WSNs that directly or indirectly affect the energy consumptions of sensor nodes have been presented. Later a survey report on several energy conserving mechanisms have been discussed in this paper. In this paper a wide range of previously proposed energy efficient solutions have been divided into six categories. Each of these categories and their example protocols along with their benefits and drawbacks are discussed here in detail. Besides the wellknown networking aspects we have explored some other factors that can also affect the energy consumptions of the network, those are the overhead energy in cluster setup phase, number of nodes in cluster and the available size of payload for data aggregations.

\section{ACKNOWLEDGEMENT}

The authors would like to acknowledge support to this work of Sir Syed University of Engineering \& Technology, Karachi, Pakistan. 


\section{REFERENCES}

Wei, L., “An Energy Aware-Based Complete Coverage and Connectivity Scheme in Clustered Wireless Sensor Network”, Sensors, Volume 9, No. 8, 2016.

Daniel,R., and Rao, K.N., “An Optimal Power Conservation Cluster Based Routing Algorithm Using Fuzzy Verdict Mechanism for Wireless Sensor Networks”, IEEE International Conference on Electrical, Electronics, Signals, Communication \&Optimization, pp. 1-9, India, 2015.

Kabara, J.,and Calle, M., "MAC Protocols used by Wireless Sensor Networks and a General Method of Performance Evaluation", International Journal of Distributed Sensor Networks, Article ID 834784, pp. 11, 2012.

Dargie, W., and Poellabauer, C., "Fundamentals of Wireless Sensor Networks: Theory and Practice”, John Wiley \& Sons, Ltd., 2010.

Huang, P.,Xiao, L., Soltani, S., Mutka, M., and Xi, N., "The Evolution of MAC Protocols in Wireless Sensor Networks: A Survey", IEEE Communications Surveys Tutorials, Volume 15, pp.101-120, 2013.

Selvi, G.V., and Manoharan, R., "Unequal Clustering Algorithm for WSN to Prolong the Network Lifetime (UCAPN)", IEEE 4th International Conference onIntelligent Systems Modeling \& Simulation, pp. 456-461, 2013.

Al Ameen, M., Islam, S.R., and Kwak, K., "Energy Saving Mechanisms for MAC Protocols in Wireless Sensor Networks”, International Journal of Distributed Sensor Networks, Volume 6, Article ID 163413, 2010.

Thangadurai, N., and Dhanasekaran, R., "Energy Efficient Cluster Based Routing Protocol for Wireless Sensor Networks”, International Journal of Computer Applications, Volume 71, pp. 43-48, 2013.

Bhakare, K.R., Krishna, R.K., and Bhakare, S. "Distance Distribution Approach of Minimizing Energy Consumption in Grid Wireless Sensor Network", International Journal of Engineering and Advanced Technology, Volume 1, No. 5, pp. 375-380, June, 2012.
[10] Mantri, D. S., Prasand, N. R., and Prasand, R., "Bandwidth Efficient Cluster-Based Data Aggregation for Wireless Sensor Network", Computers \& Electrical Engineering, Volume 41, pp. 256-264, 2015.

[11] Paul, B., and Kumar, N., "Data Gathering Protocol for Reducing Energy Utilization in a Wireless Sensor Network”, Pertanika Journal of Science \& Technology, Volume 24, No.2, 2016.

[12] Aminian, M., Akbari, M.K., and Sabael, M., "A RateDistortion Based Aggregation Method using Spatial Correlation for Wireless Sensor Networks”, Wireless Personal Communications, Volume 71, No. 3, pp. 1837-1877, 2013.

[13] Kasirajan, P., Larsen, C., and Jagannathan, S., "A New Data Aggregation Scheme via Adaptive Compression for Wireless Sensor Networks”, ACM Transactions on Sensor Networks, Volume 9, No. 1, pp. 1-26, November, 2012.

[14] Ma, Y., Guo, Y., Tian, X., and Ghanem, M., "Distributed Clustering-Based Aggregation Algorithm for Spatial Correlated Sensor Networks”, IEEE Sensors Journal, Volume11, No. 3, pp. 641-648, 2011.

[15] Tsitsipis, D., Dima, S.M., Kritikakou, A., Panagiotou, C., and Koubias, S., "Data Merge: A Data Aggregation Technique for Wireless Sensor Networks", IEEE 16th Conference on Emerging Technologies \& Factory Automation, pp.1-4, 2011.

[16] Incel, O., Ghosh, A., Krishnamachari, B., and Chintalapudi, K., "Fast Data Collection in Tree-Based Wireless Sensor Networks”, IEEE, Transactions on Mobile Computing, Volume 11, No.1, January, 2012.

[17] Tharani, C., and Ranjam, P.V., "An Energy Efficient Spatial Correlation Based Data Gathering Algorithm for Wireless Sensor Networks”, International Journal of Distributed and Parallel Systems, Volume 2, pp. 16-25, 2011.

[18] Xiang, L., Luo, J., and Vasilakos, A., “Compressed Data Aggregation for Energy Efficient Wireless Sensor Networks", 8th Annual IEEE Communications Society Conference on Sensor, Mesh and Ad Hoc Communications and Networks, pp. 46-54, 2011. 
[19] Enam, R.N., Misbahuddin, S., and Imam, M., "Energy Efficient Round Rotation Method for a Random Cluster Based WSN", IEEE International Conference on Collaboration Technologies and Systems, pp. 157-163, 2012.

[20] Yuan, H., Liu, Y., and Yu, J., “A New Energy-Efficient Unequal Clustering Algorithm for Wireless Sensor Networks”, IEEE International Conference on Computer Science and Automation Engineering, Volume1, pp. 431-434, 2012.

Karjee, J., and Jamadagni, H., "Data Accuracy Model for Distributed Clustering Algorithm Based on Spatial Data Correlation in Wireless Sensor Networks”ArXivPreprint arXiv: 1108.2644, 2011.

Zhuang,Y., Pan, J., and Wu,G., "Energy-Optimal GridBased Clustering in Wireless Micro-Sensor Networks with Data Aggregation”, International Journal of Parallel, Emergent and Distributed Systems, Volume 25, No. 6, pp. 531-550, 2010.
Enam, R.N., Qureshi, R., and Misbahuddin, S., “A Uniform Clustering Mechanism for Wireless Sensor Networks”, International Journal of Distributed Sensor Networks, Article ID: 924012, pp.1-14, 2012.

Kumar, A., Kumar, V., and Chand, N., "Energy Efficient Clustering and Cluster Head Rotation Schemefor Wireless Sensor Networks", International Journal of Advanced Computer Science and Applications, Volume 3, No. 5. 2011.

[30] Guo, W., Zhang, B., Chen, G., Wang, X., and Xiong, N., “A PSO-Optimized Minimum Spanning Tree-Based Topology Control Scheme for Wireless Sensor Networks”, International Journal of Distributed Sensor Networks, 2014.

[31] Yu,L., Wang, N., Zhang, W., and Zheng, C., "GROUP A Grid-Clustering Routing Protocol for Wireless Sensor Networks”, IEEE International Conference on Wireless Communications, Networking and Mobile Computing, pp. 1-5, 2006.

[32] Zhang, S., Fan, J., Jia, J., and Wang, J., “An Efficient Clustering Algorithm in Wireless Sensor Networks using Cooperative Communication”, International Journal of Distributed Sensor Networks, 2012.

[33] Fuad, B.I.A., “Adaptive Decentralized Reclustering Protocol for Wireless Sensor Networks", Journal of Computer SystemsScience (Science Direct), Volume 77, No. 2, 2011.

[34] Tong, M., and Tang, M., and Leach, B., “An Improved LEACH Protocol for Wireless Sensor Network", IEEE 6th International Conference on Wireless Communications Networking and Mobile Computing, pp. 1-4, 2010.

[35] Xie, D., Qi, S., Q.Z.Y.Q., and Yuan, X., “An Efficient Clustering Protocol for Wireless Sensor Networks Based on Localized Game Theoretical Approach”, International Journal of Distributed Sensor Networks, Volume 11, 2013.

[36] Afsar, M.M., "Effective Data Aggregation using a Hierarchical Multi-Layered Scheme for Large-Scale Sensor Networks”, CoRR abs/1310.8591, Volume 7, 2013. 
Mao, S., Zhao, C., Zhou, Z., and Ye, Y., “An Improved Fuzzy Unequal Clustering Algorithm for Wireless Sensor Network", Mobile Networks and Applications, Volume 18, No.2, pp. 206-214, 2013.

[38] Ye, M., Li, C., Chen, G., and Wu, J., "EECS: An Energy Efficient Clustering Scheme in Wireless Sensor Networks”, IEEE 24 ${ }^{\text {th }}$ International Conference on Performance, Computing, and Communications, pp. 535-540, 2005.

[39] Mao, Y., and Jie, W., “An Energy-Efficient Unequal Clustering Mechanism for Wireless Sensor Networks”, Proceeding of International Conference on Mobile Adhoc and Sensor System, pp.604, 2005.
[40]

Bai, F.E., Mou, H., and Sun, J., "Power-Efficient Zoning Clustering Algorithm for Wireless Sensor Networks”, IEEE International Conference on Information Engineering \& Computer Science, 2009.

[41] Vidhyapriya, R., and Thangapandian, V.P., "Energy Efficient Grid-Based Routing in Wireless Sensor Networks”, International Journal of Intelligent Computing \& Cybernetics, Volume 1, No. 2, pp. 301-318, 2008.

[42] Bahrami, S., Yousefi, H., and Movaghar, A., "DACA: Data-Aware Clustering and Aggregation in Query- Driven Wireless Sensor Networks”, IEEE 21st International Conference on Computer Communications \& Networks, pp. 1-7, 2012. 